\title{
Pharmacoepidemiology of insulin initiation in diabetes care
}

\author{
Leonard M. Pogach MD MBA
}

$\infty \infty$

See related research paper by Pérez and colleagues, page 1310

$\mathrm{M}$ any people with diabetes eventually require insulin therapy to maintain glycemic control because of progressive $\beta$-cell dysfunction. However, health care providers and patients are often reluctant to initiate insulin therapy. Some reasons for this may include clinical inertia, personal preferences or inadequate resources. This reluctance may reflect suboptimal quality of care and can be modified. ${ }^{1}$ Other reasons, such as the risk of harms of insulin therapy, may reflect rational treatment decisions for individual patients. Studies of the rates and determinants of the initiation of insulin therapy that can differentiate between appropriate and inappropriate use are needed to guide health policy and clinical practice.

In a study presented in this issue, Pérez and colleagues ${ }^{2}$ used linked prescription and claims databases to evaluate the rates and predictors of initiation of insulin therapy. A large cohort of elderly patients ( $\geq 66$ years of age) who were new users of biguanides or sulfonyureas was followed to initiation of insulin therapy, loss of drug plan eligibility or death (median follow-up of 2.9 years). The rate of insulin initiation was 9.7 cases per 1000 patient-years. Care by an endocrinologist was associated with an increased likelihood of initiating insulin therapy, as was increased illness complexity (occurrence and frequency of hospital admission in the previous year or receiving 16 or more medications), performing blood glucose monitoring, and use of medications that adversely affect glucose metabolism (e.g., steroids).

These observations appear to have clinical face validity. However, with the exception of subspecialty care, the adjusted odds ratios for the predictors were less than 1.5 , with the lower bounds of the $99 \%$ confidence interval between 1.0-1.2. The authors acknowledged that key clinical variables such as body weight, laboratory data and clinical severity of illness were not available. Thus, they could not distinguish between low rates of $\beta$-cell failure and lack of aggressive treatment.

Pérez and colleagues suggest that the rate of insulin initiation will allow for comparisons in different populations. Should administrators now consider clinical policies, such as education or increased reliance on nonphysician professionals, to increase the rate of initiation of insulin therapy? How should they interpret trends? We first need to consider both the internal validity of this study, which used a process variable as the outcome, and address concerns that should frame future studies.

Pharmacoepidemiologic studies should control for selection bias, exposure misclassification and outcome misclassification. ${ }^{3}$ In this study, although the outcome was narrowly defined, the definition of insulin initiation based on only

\begin{abstract}
Key points
- More comprehensive measures of disease burden should be used to determine whether low rates of insulin initiation reflect a lack of need or physicians' inertia.

- Unmeasured factors may influence associations between clinical and system variables and insulin initiation.

- Use of advanced statistical methods might help control for biases in future studies.
\end{abstract}

1 prescription may have overstated the rate of consistent use. However, the strength of the reported associations is subject to uncontrolled confounding. For example, exposure misclassification can result from reliance on the number of oral agents taken without knowledge of medication dosage and adherence. Chronic comorbidities can modify associations because they may represent relative contraindications to the prescription of either oral agents or insulin because of altered metabolism (e.g., hepatic or renal disease), appetite (e.g., gastrointestinal disorders), frailty or cognitive function.

Evaluation of potential biases from unmeasured variables such as these will likely require more advanced methods. ${ }^{3}$ Propensity analyses can balance comparison groups by matching patients based on demographics, comorbid illnesses, laboratory values and medications, which would allow different intensities of oral agents or insulin to be compared as if the treatment assignment had been randomized. ${ }^{4}$ Even so, confounding can occur because of other unmeasured variables, such as social support or inability to follow medical advice, which could be reasonable exclusion criteria yet could lead to lower rates of insulin initiation.

Nonclinical confounding variables may also be relevant. For example, some primary care physicians may not have adequate support staff. Thus, an alternative approach would be to define an instrumental variable linked to the treatment but not the outcome, such as the availability of endocrinologists (i.e., patients with greater access to subspecialty care would be more likely to receive insulin). Finally, analysis with techniques such as the Heckman model can control for selfselection biases, such as enrollment into managed care or feefor-service. However, this may not be of importance in universal health care systems.

Even if such analyses yielded similar results, thus increasing confidence in the estimates of insulin initiation by Pérez

Leonard Pogach is with the Department of Veterans Affairs, New Jersey Health Care System, East Orange, and the University of Medicine and Dentistry of New Jersey, New Jersey Medical School, Newark, USA. 
and colleagues, evaluation of additional outcomes will be essential to direct policy. First, appropriate levels of glycemic control in elderly people must be defined. Although the 2003 Canadian Diabetes Association guidelines recommended tight control in "most persons" with type 2 diabetes, published guidelines in the United States have recommended individualization of targets based on life expectancy, comorbid conditions and shared decision-making. ${ }^{5}$

Second, appropriate clinical judgment may be a reasonable explanation for the study findings. The risks of insulin use in elderly patients are well known. In the United States, insulin is the second most common drug associated with emergency department visits and hospital admissions. ${ }^{6}$ In Canada, insulin monotherapy and combined oral therapy have been reported to be associated with automobile accidents. ${ }^{7}$ Third, intensive treatment that relies on insulin may be associated with increased cardiovascular mortality. ${ }^{8,9}$ Thus, monitoring for excess mortality as well as episodes of hypoglycemia and other potential adverse outcomes must accompany any evaluation of glycemic control after insulin initiation. Fourth, longitudinal modelling of hemoglobin $\mathrm{A}_{1 \mathrm{c}}$ in fixed cohorts can minimize biases inherent in estimating trends from cross-sectional data. ${ }^{10}$ Finally, total costs should be evaluated, but only in the context of overall health outcomes.

How, then, should we interpret the findings of the current study? The results provide a signal, albeit of uncertain strength, that there could be underuse of insulin among elderly patients. However, the initial recommended course of action should not be aggressive use of insulin to control glycemia, but rather an attempt to identify cohorts for whom failure of insulin therapy would be an obvious quality issue, such as those with higher hemoglobin $\mathrm{A}_{\mathrm{lc}}$ levels or hospital admissions for inadequate glycemic control. ${ }^{11}$ Well-conducted analyses of data sets enriched with the clinical variables previously described may enable simultaneous evaluation of quality, safety, outcomes and costs in multiple cohorts of elderly peoples. ${ }^{11}$ The current study is a step in that direction, but much distance must be travelled before we can state that pharmacoepidemiologic research has kept its promise to provide policy-makers with comprehensive information to understand both the benefits and risks of insulin therapy in elderly patients.

\section{Competing interests: None declared.}

The views expressed in this commentary are solely those of the author and do not reflect the views of the Department of Veterans Affairs or the affiliate institution.

Acknowledgement: I thank Donald R. Miller ScD for reviewing and providing feedback on this commentary.

\section{REFERENCES}

1. Peyrot M, Rubin RR, Lauritzen T, et al.; International DAWN Advisory Panel. Resistance to insulin therapy among patients and providers: results of the cross-national Diabetes Attitudes, Wishes, and Needs (DAWN) study. Diabetes Care 2005;28:2673-9.

2. Pérez N, Moisan J, Sirois C, et al. Initiation of insulin therapy in elderly patient taking oral antidiabetes drugs. CMAJ 2009;180:1310-6.
3. Pizer SD. An intuitive review of methods for observational studies of comparative effectiveness. Health Serv Outcomes Res Methodol 2009;9:54-68.

4. Shah BR, Hux JE, Laupacis A, et al. Clinical inertia in response to inadequate glycemic control: Do specialists differ from primary care physicians? Diabetes Care 2005;28:600-6.

5. Qaseem A, Vijan S, Snow V, et al.; Clinical Efficacy Assessment Subcommittee of the American College of Physicians. Glycemic control and type 2 diabetes mellitus: the optimal hemoglobin A1c targets. A guidance statement from the American College of Physicians. Ann Intern Med 2007;147:417-22.

6. Budnitz DS, Shehab N, Kegler SR, et al. Medication use leading to emergency department visits for adverse drug events in older adults. Ann Intern Med 2007; 147:755-65.

7. Hemmelgarn B, Lévesque LE, Suissa S. Anti-diabetic drug use and the risk of motor vehicle crash in the elderly. Can J Clin Pharmacol 2006;13:e112-20.

8. Gerstein HC, Miller ME, Byington RP, et al.; Action to Control Cardiovascular Risk in Diabetes Study Group. Effects of intensive glucose lowering in type 2 diabetes. N Engl J Med 2008;358:2545-59.

9. Duckworth W, Abraira C, Moritz T, et al.; VADT Investigators. Glucose control and vascular complications in veterans with type 2 diabetes. $N$ Engl J Med 2009;360:129-39.

10. Thompson W, Wang H, Xie M, et al. Assessing quality of diabetes care by measuring longitudinal changes in hemoglobin A1c in the Veterans Health Administration. Health Serv Res 2005;40:1818-35.

11. Helmer DA, Tseng CL, Rajan M, et al. Can ambulatory care prevent hospitalization for metabolic decompensation? Med Care 2008;46:148-57.

Correspondence to: Dr. Leonard Pogach, Department of Veterans Affairs, New Jersey Health Care System, 385 Tremont Ave.,

East Orange, NJ 07018 USA; fax 973 395-7111;

leonard.pogach@va.gov

\section{Send us your perspective -} we like controversy.

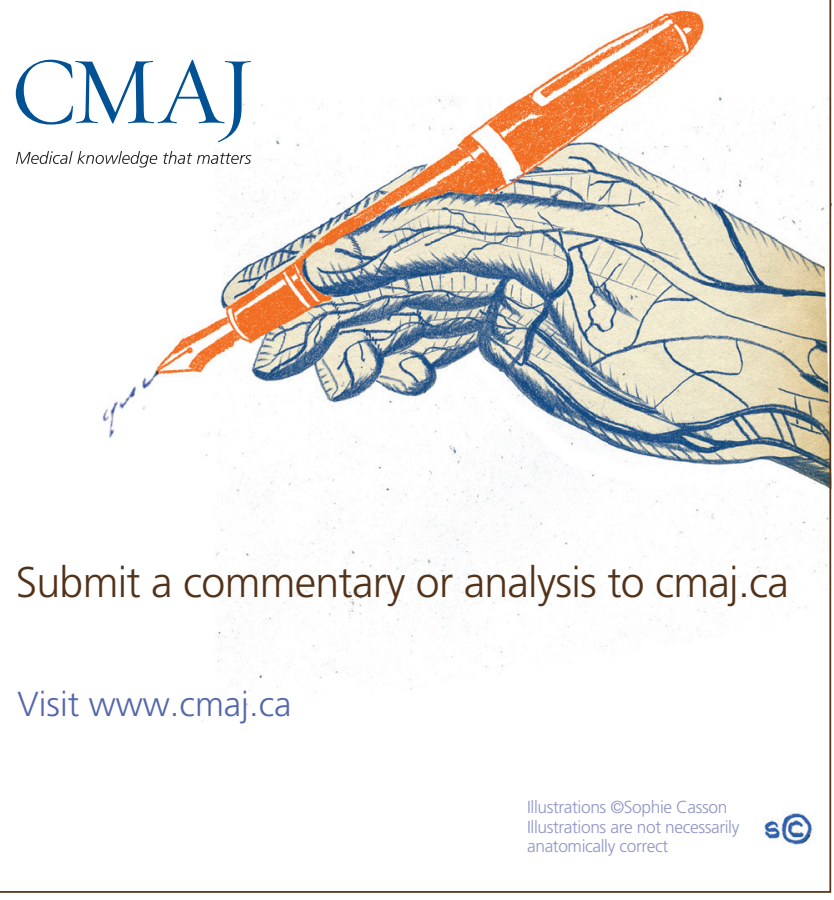

